\title{
4D-CT parathyroid increases the likelihood of localising parathyroid adenoma (PA) in patients with primary hyperparathyroidism (PHPT) and indeterminate Tc99m-Sestamibi+1-123 subtraction scan
}

Brian C Lee ${ }^{1}$, Manish Pandit ${ }^{2}$ and Ansu Basu ${ }^{1}$

${ }^{1}$ Department of Diabetes, Endocrinology and Lipid Metabolism, ${ }^{2}$ Department of Nuclear Medicine, Sandwell and West Birmingham Hospitals NHS

Trust, Birmingham , UK. B18 7QH

\section{Introduction:}

Tc99m-Sestamibi+l-123 subtraction using planar and SPECT-CT is commonly used to investigate PHPT. Our centre previously published sensitivity and specificity of $92 \%$ and $86 \%$ respectively for Tc99m-Sestamibi+l-123 subtraction. Five false-negative studies required neck exploratory surgery. One false-positive study incorrectly localised a PA which was not found in surgery ( $n=67)$. In recent years 4D-CT parathyroid has emerged as a useful technique to detect and localise PA in the work-up of patients with PHPT. 4D$\mathrm{CT}$ is a specialised imaging which involves the administration of an iodine-based contrast to enhance the quality of imaging. Images are acquired with the CT scanner at very specific time when the contrast is maximally taken up by the parathyroid glands. The 'fourth dimension' is time. We have been using 4D-CT as a second line investigation following an indeterminate Tc99m-Sestamibi+l-123 subtraction.

\section{Description of methods/design:}

We retrospectively reviewed case records of patients with surgically proven PA who had also 4D-CT post subtraction scan. A common cause of indeterminate Tc99m-Sestamibi+l-123 subtraction is the confounding factor of multi-nodular goitre or nodules.

Demographic data (age, sex, pre and postoperative calcium/PTH) were presented below. Histopathology of surgical specimens was also examined. Sensitivity and specificity of 4D-CT was calculated with surgically proven PA as the reference standard.

\section{Results:}

$\mathrm{N}=8, \mathrm{M}: \mathrm{F}=2: 6$, Age $45-72$ years.

All Calcium decreased postoperatively except 2 patients (One 4D-CT positive and 1 normal) in whom Calcium/PTH remained higher than normal with surgical specimen confirmed normal parathyroid tissue . In patients with surgically proven PA, 4D-CT parathyroid performed pre-operatively demonstrated a sensitivity of $83 \%$ and a positive predictive value of $83 \%$.

\begin{tabular}{|c|c|c|c|c|}
\hline & Parathyroid adenoma (+ve) & Parathyroid adenoma (-ve) & \\
\hline 4D-CT (+ve) & 5 & 1 & 6 & Positive Predictive Value 5/(5+1) $\mathbf{8 3} \%$ \\
\hline 4D-CT (-ve) & 1 & 1 & 2 & \\
\hline & 6 & 2 & 8 & \\
\hline
\end{tabular}

\section{Conclusion:}

In a cohort of patients with indeterminate subtraction scans for investigation of PHPT, 4D-CT parathyroid significantly improved the diagnostic value for pre-operative localisation. It is therefore the preferred second line investigation of choice, with high sensitivity and predictive value allowing accurate pre-operative localisation for minimally invasive parathyroidectomy. The patients can also be better informed of the likelihood of finding of PA prior to surgery.
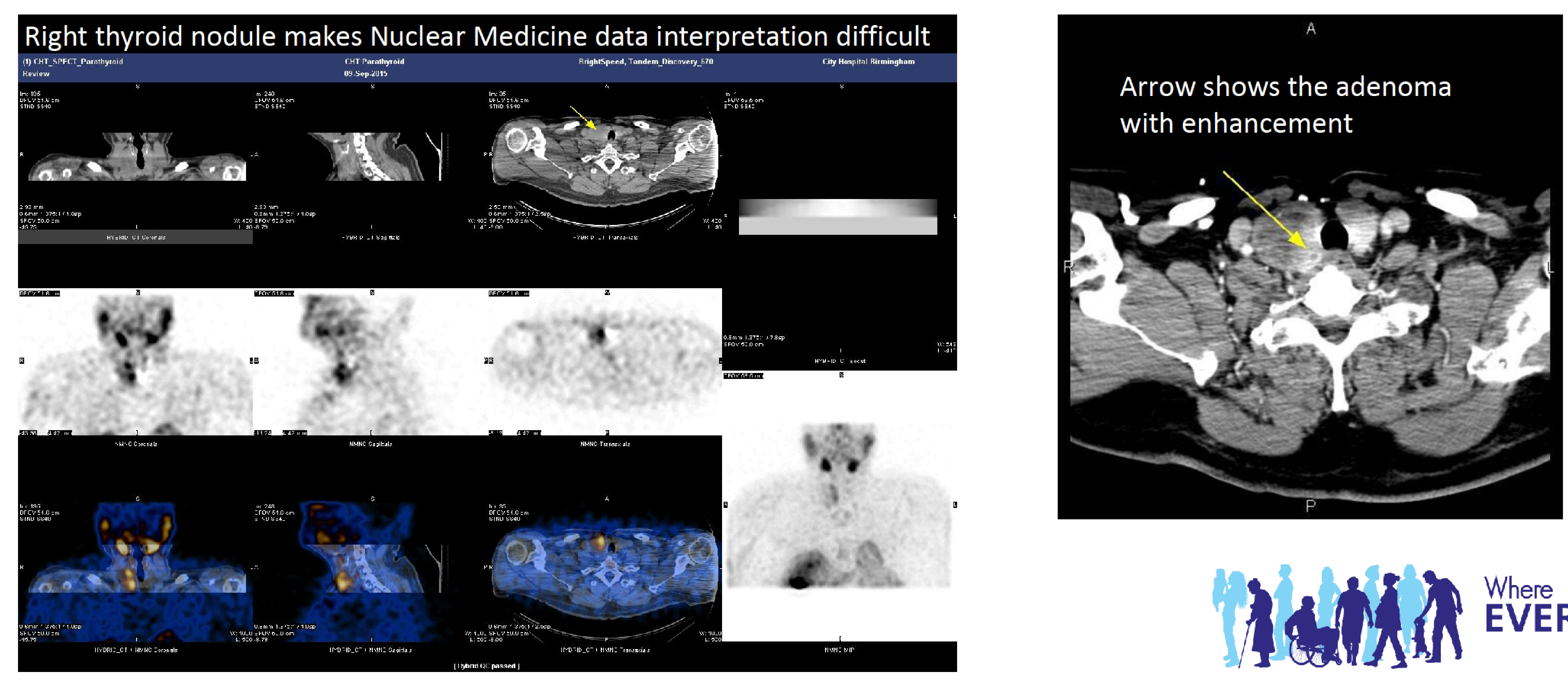